\title{
Is it time to Stop Layering Ceramics?
}

\author{
Ahmed Abyad* \\ Department of Oral Rehabilitation Sciences, Lebanon
}

*Corresponding author: Ahmed Abyad, Senior Lecturer of Dental Biomaterials, Department of Oral Rehabilitation Sciences, Beirut Arab University, Beirut, Lebanon.

\begin{abstract}
Layering ceramics is a technique utilized by majority of dental technicians around the world. This technique was long used and has done service to the dental community; However, with the increasing use of monolithic restorations, layered restorations are proving to be less effective. Is it time for technicians to stop using the layering brushes?
\end{abstract}

\section{Short Communication}

The esthetics of anterior ceramics are driven by two factors, symmetry and thickness. To the surprise of many, color plays a minimal role; an even greater surprise is that layering plays no role in the esthetic outcome of the restorations. In fact, in most cases layering negatively impacts the esthetic potential of a restoration by causing imbalances with neighboring teeth in addition to leading in increased thickness of the restoration. Remember, if the dentists prepares a tooth by $0.7 \mathrm{~mm}$, and the technician provides a $1.5 \mathrm{~mm}$ restoration, there will be $0.8 \mathrm{~mm}$ extra ceramics in the transverse plane. This means an extra tooth exists on over the original tooth. To layer, a dental technician has to use a ceramic brush and add ceramic material layer by layer. Due to this process being highly dependent on the technician's skills, the esthetics of layered ceramics becomes a matter of subjectivity and chance. In many cases the restorations lack the balance and symmetry required especially in cases of central incisors where the symmetry plays a crucial role in determining the esthetic outcome. The other problem which accompanies layering is the need for thickness.

Dentists learn in dental school minimal invasive principles and how to operate removing as little of tooth structure as possible. On the other hand, technicians who layer favor heavily prepared teeth which allow room for layering of the ceramic. This creates the dilemma of over contoured restorations where the majority of "Hollywood smiles" are turning out to be bulky sets of teeth with dark contacts. In fact, in most cases layering negatively impacts the esthetic potential of a restoration by causing imbalances with neighboring teeth in addition to leading in increased thickness of the restoration. Remember, if the dentists prepares a tooth by 0.7 $\mathrm{mm}$, and the technician provides a $1.5 \mathrm{~mm}$ restoration, there will be $0.8 \mathrm{~mm}$ extra ceramics in the transverse plane; This means an extra tooth exists over the original tooth.

The questions which got to be answered, can the outcome attained by layering justify the thickness of the restoration? Can few mamelons on the incisal third of the restoration justify thicknesses which in many cases exceed one and a half millimeters?

The clinician's goal should be providing natural restorations which satisfy the patient expectations. Thick restorations are not natural; they can be even noticed by the untrained eye. On the contrary, mamelons require trained eyes to be noticed. In fact, to show their work, clinicians utilize DSLRS, macro lenses, dual flashes and many more equipment. In regular day light these mamelons and characterizations created by layering mean nothing to the general public.

This is why dental technicians should leave the brush and use alternative techniques which they already have and use. Monolithic restorations can provide the utmost esthetics due to their minimal thickness. These restorations will also exhibit the highest mechanical properties due the absence of layers inside. Layered ceramics are notorious for delamination and ceramic chipping and this is highly unlikely with monolithic restorations. Surface characterization by introducing tertiary anatomy in addition to the use of staining glazes can result in highly esthetic restorations which at the time same time exhibit high longevity. Remember, the age of the restorations is governed by the weakest part of the ceramic and not the strongest. If the porcelain of 100 MPA is layered over a 
zirconia coping of $1000 \mathrm{MPa}$, the flexural strength of your crown is still $100 \mathrm{MPa}$. This it is because when the layering porcelain chips in the anterior region it has to be replaced.

As for those who layer in the posterior region in single crowns or three unit bridges, to them it should be clear they are practicing the lowest quality of dentistry available in 2018. There is no excuse to weaken a posterior crown by layering just for the sake of selfsatisfaction.

With time, all technicians are going to leave the brush, probably just using it for glazing purposes. Layering will be ancient technique in few years and to my fellow young technicians, I invite them to master design and characterization of monolithic restorations, and to stop wasting their time learning techniques which will go extinct. When CAD CAMs becomes available to all dentists in the future, they will want technicians who can operate these units better than them, and to be a successful, they have to prepare when this time comes.

\section{Acknowledgment}

None.

\section{Conflict of Interest}

No conflict of interest. 